262 18. Oetting er, Summenrechmung der durch einfüche Functionen erzeuglen Reihen.

\title{
18. \\ Summenrechnung der durch einfache Functionen erzeugten Reihen.
}

(Von dem Herrn Prof. Oettinger zu Heidelberg.)

(Fortsetzung von No.6. und 14. Band XI., No. 24; Band XII., und No. 22., 23. und 24. Band XIII.)

\section{$A$. \\ \$. 72.}

Die bisherigen Untersuchungen über Aufstufungen und Unterschiede einfacher und zusammengesetzter Functionen sind als Vorbereitungen zur Aufsuchung weiterer Resultate zu betrachten und erhalten daher ihre weitere Bedeutung in der Eigenschaft: Anwendungen aus sich ziehen zu lassen, die wir auf anderem Wege des Calculs entweder gar nicht oder nicht so zweckmälsig gewinnen können. Besonders zweckmälsig lassen sie sich zur Summirung solcher Reihen gebrauchen, die durch Functionen, deren Aufstufungen und Unterschiede leicht gebildet werden können, erzeugt werden.

Wir versuchen es nun, die Summen der durch die Analysis bekannten Functionen nach den bisherigen Vorbereitungen aufzusuchen, und sie in einem Zusammenhange darzustellen, und wünschen, dafs diese Arbeit als ein Versuch, diesen $Z_{w e i g}$ der Wissenschaft zu bearbeiten betrachtet und günstig aufgenommen werden möge.

Wir beschäftigen uns zu dem Ende zuerst mit der Summirung der durch einfache Functionen erzeugten Reihen; dann mit der Summirung derer, die durch zusammengesetzte erzeugt werden.

Bei allen Summenreihen lassen sich leicht zwei Fälle unterscheiden. Es sind nämlich die Glieder der Reihen mit einerlei (positiven), oder abwechselnden (positiven oder negativen) Zeichen versehen. Beide Arten sollen im Folgenden betrachtet werden. Zu der Darstellung der Summen der durch einfachęn Functionen erzeugten Reihen wenden wir uns zuerst. 


\section{§. 73.}

Die Gleichungen, die wir in $\$ .4$. bei der Darstellung der negativen Aufstufungen aus $(n+1)$ Gliedern der Grundreihe, und \$. 25. für die der negativen Unterschiede aus gleicher Glieder-Anzabl gefunden haben, sind, wie man sich leicht überzeugt, von der Beschaffenheit, dafs sie Summenreihen bilden, die durch einfache Functionen erzeugt werden, nach einem bestimmten Gesetze fallen oder steigen, und zwar so, dafs die negativen Aufstufungen Summenreihen mit abwechselnden Zeichen, die negativen Unterschiede Summenreihen mit einerlei Zeichen bilden. Die Richtigkeit dieser Behauptung verdeutlicht sich sehr dadurch, dafs wir die erste negative Aufstufung und den ersten negativen Unterschied, wie sie aus $(n+1)$ Gliedern der Grundreihe gebildet werden, in folgender Anordnung betrachten. Die Gleichung (18.) giebt folgende Darstellung :

$$
\boldsymbol{X}_{-1}-\boldsymbol{X}_{-2}+\boldsymbol{X}_{-3}-\boldsymbol{X}_{-4}+\ldots(-)^{n} \boldsymbol{X}_{-n-1}=\zeta^{-1} \boldsymbol{X}_{0}(-)^{n} \zeta^{-1} \boldsymbol{X}_{-n-1} \text {, }
$$

die Gleichung (123.) folgende:

$$
X_{-1}+X_{-2}+X_{-3}+X_{-4}+\ldots .+X_{-n-1}=\Delta^{-1} X_{0}-\Delta^{-1} X_{-n-1} \text {. }
$$

Die Ausdrücke auf der linken Seite in den beiden Gleichungen erscheinen als Glieder einer Summenreibe, die einem bestimmten Gesetze unterliegen. Die auf der rechten Seite sind die Summen-Ausdrücke, die ihnen zugehören. Nimmt man nun für $X$ irgend eine Function an, so wird man nach Maafsgabe der vorstehenden Gleichungen die veränderliche Grölse in ihr abnehmen lassen und von der Function $X_{1}$ und $X_{-n-1}$ die ersten negativen Aufstufungen, oder die ersten negativen Unterschiede suchen und sie nach der Vorschrift der Zeichen verbinden müssen, um die Summen-Ausdrücke der Reihen, die mit abwechselnden oder mit einerlei Zeichen verbunden sind, darstellen zu können.

Nach diesen Bemerkungen fällt also die Zusammenrechnung der Functionen mit der Darstellung der negativen Aufstufungen und der negativen Unterschiede zusammen; denn letztere bedingen die Summen-Ausdrücke summirbarer Reihen.

Wir beschränken uns aber nicht auf die zwei vorliegenden speciellen Fälle, sondern gehen von den allgemêinern Gleichungen (25.) und (125.) aus, und beginnen mit den negativen Unterschieden, weil diese auf die Summirung soleher Reihen führen, deren Glieder mit einerlei Zeichen verbunden sind. Aus (126.) entnehmen wir: 
264 18, Oetting er, Summenrechnung der durch einfache Functionen er:zeugten Reihen.

$$
\text { 345. } \begin{gathered}
\quad X_{-r}+\frac{2^{r-1 \mid 1}}{1^{r-1 \mid 1}} X_{-r-1}+\frac{3^{r-1 \mid 1}}{1^{r-1 \mid 1}} X_{-r-2}+\ldots \ldots+\frac{(n+1)^{r-1 \mid 1}}{1^{r-1 \mid 1}} X_{-n-r} \\
=\Delta^{-r} X_{0}-\frac{(n+1)^{r-1 \mid 1}}{1^{r-1 \mid 1}} \Delta^{-1} X_{-n-r}-\frac{(n+1)^{r-2 \mid 1}}{1^{r-2 \mid 1}} \Delta^{-2} X_{-n-r+1}-\ldots \\
\ldots \ldots-\frac{n+1}{1} \Delta^{-r+1} X_{-n-2}-\Delta^{-r} X_{-n-1} 5
\end{gathered}
$$

aus (25.) Folgendes:

346. $\quad X_{-r}-\frac{2^{r-1 \mid 1}}{1^{r-1 \mid 1}} X_{-r-1}-\frac{3^{r-1 \mid 1}}{1^{r-1 \mid 1}} X_{-r-2}+\ldots \ldots(-)^{n(n+1)^{r-1 \mid 1}} X_{-x-r}$

$$
\begin{aligned}
=\zeta^{-r} X_{0}(-)^{r}\left[\frac{(n+1)^{r-1 \mid 1}}{1^{r-1 \mid 1}} \zeta^{-1} X_{-n-r}\right. & +\frac{(n+1)^{r-2 \mid 1}}{1^{r-2 \mid 1}} \zeta^{-2} X_{-n-r+1}+\ldots \\
& \left.\cdots \cdot+\frac{n+1}{1} \zeta^{-r+1} X_{-n-2}+\zeta^{-r} X_{-n-1}\right] .
\end{aligned}
$$

Man erkennt leicht die Ûbereinstimmung und Verschiedenheit in den Grundzigen der Bildungsweise der vorliegenden Gleichungen. Vorzahlen und Stellenzahlen sind vollkommen dieselben;. Zeichen und Geschäfte, die mit den Functionen vorgenommen werden sollen, sind verschieden.

Von den Gliedern der Summenreihe hängt die Gestalt der summirbaren Reihe ab. Nur diejenigen Reihen, welche dem angegebenen Grundgesetze unterliegen, sind nach diesen Gleichungen summirbar. Dies ist schọn längst als Kennzeichen summirbarer Reihen anerkannt.

Die Reihen sind aber in der yorliegenden Form nicht brauchbar. Um sie brauchbar zu machen, erhühen wir die Ștellenzahlen aller Glieder dieser Gleichungen um $n+r$, und erhalten aus (345.)

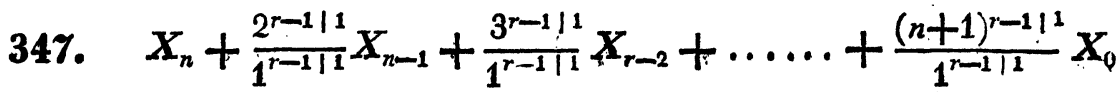

$$
\begin{aligned}
& =\Delta^{-r} X_{n+r}-\frac{(n+1)^{r-1 \mid 1}}{1^{r-1 \mid 1}} \Delta^{-1} X_{0}-\frac{(n+1)^{r-2 \mid 1}}{1^{r-2 \mid 1}} \Delta^{-2} X_{1}-\ldots \\
& \cdots-\frac{n+1}{1} \Delta^{-r+1} X_{r-2}-\Delta^{-r} X_{r-1} \text {. }
\end{aligned}
$$

Bei der Gleichung (346.) ist jedoch zu berïksichtigen, dafs sich die Form des Summen- Ausdruckes ändert, je nachdem $n$ eine gerade oder ungerade Zahl ist. Ist $n$ eine gerade Zahl, so ist $(-)^{r}=+$ und es erscheinen alle Glieder im Sammen-Aus,drucke mit dem positiven Zeichen. Ist $n$ eine ungerade $\mathrm{Zahl}$, so wird $(-)^{n}=-$, und es erscheinen alle in Klammern eingeschlossenen Glieder des Summen-Ausdruckes negativ. Hiernach erhalten wir zwei verschiedene Darstellungen. Für ein gerades $n$, oder einẹ ungerade Gliederanzabl: 
18. Oetting er, Summenrechnung der durch einfache Functionen erzeugten Reihen. 265

348. $X_{n}-\frac{2^{r-1 \mid 1}}{1^{r-1 \mid 1}} X_{n-1}+\frac{3^{r-1 \mid 1}}{1^{r-1 \mid 1}} X_{n-2}-\ldots \ldots+\frac{(n+1)^{r-1 \mid 1}}{1^{r-1 \mid 1}} X_{0}$

$=\zeta^{-r} X_{n+r}+\frac{(n+1)^{r-1 \mid 1}}{1^{r-1 \mid 1}} \zeta^{-1} X_{0}+\frac{(n+1)^{r-2 ! 1}}{1^{r-2 ! 1}} \zeta^{-2} X_{1}+\cdots+\xi^{-r} X_{r-1}$,

und futr ein ungerades $n$ oder eine gerade Glieder-Anzahl s

349. $X_{n}-\frac{2^{r-1 \mid 1}}{1^{r-1 \mid 1}} X_{n-1}+\frac{3^{r-1 \mid 1}}{1^{r-1 \mid 1}} X_{n-2}+\ldots \ldots-\frac{(n+1)^{r-1 \mid 1}}{1^{r-1 \mid 1}} X_{0}$

$=\zeta^{-r} X_{n+r}-\frac{(n+1)^{r-1 \mid 1}}{1^{r-1 \mid 1}} \zeta^{-1} X_{0}-\frac{(n+1)^{r-2 \mid 1}}{1^{r-2 \mid 1}}-\ldots-\zeta^{-r} X_{r-1}$.

Zur bequemeren Benutzung dieser allgemeinen Gleichungen geben wir hier eine Zusammenstellung einiger aus ihnen entnommenen speciellen Fälle. Aus (347.) wird, wenn statt $r$ allmälig die Werthe 1, 2, 3, . . e eingeführt werden und mit dem mindesten Gliede $X_{0}$ der Summenreihe begonnen wird :

350.

$$
\left\{\begin{array}{c}
X_{0}+X_{x}+X_{2}+X_{3}+\ldots \ldots \ldots+X_{n}=\Delta^{-1} X_{n+1}-\Delta^{-1} X_{0}, \\
(n+1) X_{0}+n . X_{x}+(n-1) X_{2}+\ldots+2 X_{n-1}+X_{n} \\
=\Delta^{-2} X_{n+2}-\frac{n+1}{1} \Delta^{-1} X_{0}-\Delta^{-2} X_{x}, \\
\frac{(n+1)(n+2)}{1.2} X_{0}+\frac{n(n+1)}{1.2}+\ldots \ldots \ldots \frac{3.2}{1.2} X_{n-1}+X_{n} \\
=\Delta^{-3} X_{n+3}-\frac{(n+1)(n+2)}{1.2} \Delta^{-1} X_{0}-\frac{n+1}{1} \Delta^{-2} X_{x}-\Delta^{-3} X_{2}, \\
\frac{(n+1)(n+2)(n+3)}{1.2 .3} X_{0}+\frac{n(n+1)(n+2)}{1.2 .3}+\ldots \ldots \cdot \frac{4.3 .2}{1.2 .3} X_{n-1}+\frac{3.2 .1}{1.2 .3} X_{n} \\
=\Delta^{-4} X_{n+4}-\frac{(n+1)(n+2)(n+3)}{1.2 .3} \Delta^{-1} X_{0}-\frac{(n+1)(n+2)}{1.2} \Delta^{-2} X_{2}-\frac{n+1}{1} \Delta^{-2} X_{2}-\Delta^{-3} X_{3} \\
\text { u. s. w. }
\end{array}\right.
$$

Aus (348.) erhalten wir folgende Fälle für ein gerades $n$ :

$$
351 .\left\{\begin{array}{c}
X_{0}-X_{1}+X_{2}-X_{3} \ldots \ldots \ldots .+X_{n}=\zeta^{-1} X_{n+1}+\zeta^{-1} X_{0}, \\
(n+1) X_{0}-n X_{1}+(n-1) X_{2}-\ldots \ldots+X_{n}=\zeta^{-2} X_{n+2}+\frac{n+1}{1} \zeta^{-1} X_{0}+\zeta^{-2} X_{1}, \\
\frac{(n+1)(n+2)}{1.2} X_{0}-\frac{n(n+1)}{1.2} X_{1}+\frac{(n-1) n}{1.2} X_{2}-\ldots .-\frac{3.2}{1.2} X_{n-1}+X_{n} \\
=\zeta^{-3} X_{n+3}+\frac{(n+1)(n+2)}{1.2} \zeta^{-1} X_{0}+\frac{n+1}{1} \zeta^{-2} X_{1}+\zeta^{-3} X_{2}, \\
\frac{(n+1)(n+2)(n+3)}{1.2 .3} X_{0}-\frac{n(n+1)(n+2)}{1.2 .3} X_{2}+\frac{(n-1) n(n+1)}{1.2 .3} X_{2}-\ldots . .-\frac{4.3 .2}{1.2 .3} X_{n-1}+X_{n} \\
=\zeta^{-4} X_{n-4}+\frac{(n+1)(n+2)(n+3)}{1.2 .3} \zeta^{-1} X_{0}+\frac{(n+1)(n+2)}{1.2} \zeta^{-2} X_{1}+\frac{n+1}{1} \zeta^{-3} X_{2}+\zeta^{-4} X_{3} \\
\text { u. s. w. }
\end{array}\right.
$$

Crelle's Journal d. M. Bd. XIV. Hft. 3. 
266 18. Oetting er, Summenrechntung der durch einfache Functionen erzeugten Reihen.

Um aus der allgemeinen Gleichung (349.) ähnliche Reihen abzuleiten, die mit dem mindestén Gliede $X_{\overline{0}}$, als einem positiven, und mit $X_{n}$ als höchsten enden, müssen die Zeichen der ganzen Gleichung in die entgegengesetzten verwandelt werden. Dadurch entstehen folgende Entwicklungen für ein ungerades $n$ :

352. $\left\{\begin{array}{c}X_{0}-X_{1}+X_{2}-X_{3}+\ldots-X_{n}=-\zeta^{-1} X_{n+1}+\zeta^{-1} X_{0}, \\ (n+1) X_{0}-n X_{1}+(n-1) X_{2}-\ldots+2 X_{n-1}-X_{n} \\ =-\zeta^{-2} X^{n+2}+\frac{n+1}{1} \zeta^{-1} X_{0}+\zeta^{-2} X_{1}, \\ \frac{(n+1)(n+2)}{1.2} X_{0}-\frac{n(n+1)}{1.2} X_{1}+\ldots+\frac{3.2}{1.2} X_{n-1}-\frac{2.1}{1.2} X_{n} \\ =-\zeta^{-3} X_{n+3}+\frac{(n+1)(n+2)}{1.2} \zeta^{-1} X_{0}+\frac{n+1}{1} \zeta^{-2} X_{1}+\zeta^{-3} X_{2}, \\ \frac{(n+1)(n+2)(n+3)}{1.2 .3} X_{0}-\frac{n(n+1)(n+2)}{1.2 .3} X_{1}+\frac{(n-1) n(n+1)}{1.2 .3} X_{2}-\ldots+\frac{4.3 .2}{1.2 .3} X^{n-1}-X_{n} \\ =-\zeta^{-4} X_{n+4}+\frac{(n+1)(n+2)(n+3)}{1.2 .3} \zeta^{-1} X_{0}+\frac{(n+1)(n+2)}{1.2} \zeta^{-2} X_{1}+\frac{n+1}{1} \zeta^{-3} X_{2}+\zeta^{-4} X_{3} \\ \text { u. s. w. }\end{array}\right.$

Die hier gegebenen Zusammenstellungen zeigen, dafs die auf sie gegründete Summirungsmethode abhängt von der Bestimmung der negativen Unterschiede und Aufstufungen der Functionen. Wie diese aufgefunden werden könrren, haben wir gezeigt. Die zu suchenden Summenausdrücke werden sich immer bestimmen lassen, wenn sich die negativen Unterschiede und Aufstufungen bilden lassen.

Zugleich geht aus der gefundenen Darstellung hervor, dafs die auf sie gegründete Summirungsmethode allgemein ist, und von allen Functionen ohne Unterschied gilt. Daher lassen sich durch sie, wie wir sehen werden, auch alle Funotionen summiren, wenn auch nicht alle bis jetzt summirt wurden.

Die Grundreihe, die allen diesen Entwickelungen zu Grunde liegt, ist :

$$
X_{0}, \quad X_{1}, X_{2}, \ldots X_{n} \text {. }
$$

Sie läfst sich auch, wenn $X=f x$ und $\Delta x$ die Zunahme ist, 80 darstellen:

$$
f x, f(x+\Delta x), f(x+2 \Delta x), \ldots f(x+n \Delta x) \text {. }
$$

Nach diesen Bemerkungen gehen, wir nun zu der Darstellung der einzelnen Summenreiben mit ihren Summen-Ausdrücken über, und betrachten zuerst die mit gleichen, dann die mit abwechselnden Zeichen 
18. Oeltinger, Summenrechnung der durch einfache Functionen er zeugten Reihen. 267

Summenreihen und Summen-Ausdrücke der Potenzialfunctionen.

s. 74.

Summirung der Potenzenreihen, deren Glieder mit positiven Zeichen verbunden sind.

Um die Summe der Potenzenreihe, deren Glieder mit einerlei Zeichen verbunden sind, zu gewinnęn, legen wir die erste Reihe (350.)

$$
X_{0}+X_{1}+X_{2}+\ldots .+X_{n}=\Delta^{-1} X_{n+1}-\Delta^{-1} X_{0}
$$

zu Grunde, und setzen in ihr $X_{0}=x^{p}$, dann wird, $X_{1}=(x+\Delta x)^{p}$, $X_{2}=(x+2 \Delta x)^{p}, \quad X_{n}=(x+n \Delta x)^{p}$ und $X_{n+1}=(x+(n+1) \Delta x)^{p}$. Hieraus entsteht, durch Einführung dieser Werthe:

$x^{p}+(x+\Delta x)^{p}+(x+2 \Delta x)^{p}+\ldots .+(x+n \Delta x)^{p}=\Delta^{-1}(x+(n+1) \Delta x)^{p}-\Delta^{-1} x^{p}$, die Bestimmung des Summen-Ausdrucks hängt von der Darstellung der beiden negativen Unterschiede von $(x+(n+1) \Delta x)^{p}$ und $x^{p}$ ab. Diese gewinnen sich leicht, wenn in der Gleichung (192.) $x+(n+1) \Delta x$ statt $\boldsymbol{y}$ und $x$ statt $y$ gesetzt wird. Hieraus folgt unmittelbar:

$$
\begin{array}{ll}
x^{p}+(x+\Delta x)^{p}+(x+2 \Delta x)^{p} & +\cdots+(x+n \Delta x)^{p} \\
=\frac{(x+(n+1) \Delta x)^{p+1}}{(p+1) \Delta x} & -\frac{x^{p+1}}{(p+1) \Delta x} \\
-\frac{1}{2}(x+(n+1) \Delta x)^{p} & +\frac{x^{p}}{2} \\
+\frac{1}{6} \cdot \frac{1}{2} \cdot \frac{p}{1}(x+(n+1) \Delta x)^{p-1} \Delta x & -\frac{1}{6} \cdot \frac{p}{2} x^{p-1} \Delta x \\
-\frac{1}{30} \cdot \frac{1}{4} \cdot \frac{p(p-1)(p-2)}{1.2 .3}(x+(n+1) \Delta x)^{p-3}(\Delta x)^{3}+\frac{1}{30} \cdot \frac{1}{4} \cdot \frac{p(p-1)(p-2)}{1.2 .3} x^{p-3}(\Delta x)^{2}
\end{array}
$$

Wir bemerken, dafs in dieser Gleichung das höchste Glied der Summenreihe $(x+n \Delta x)^{p}$ mit dem Ausdrucke $(x+(n+1) \Delta x)^{p}$, im SummenAusdrucke, der Form nach nicht harmonirt. Zählen wir dieses Glied auf beiden Seiten zu, scheiden dann die in beiden Reihen gemeinschaftlichen Factoren aus, so gewinnen wir folgende Darstellung

353. $x^{p}+(x+\Delta x)^{p}+(x+2 \Delta x)^{p}+\ldots . .+(x+(n+1) \Delta x)^{p}$

$$
\begin{aligned}
& =\frac{1}{(p+1) \Delta x}\left[(x+(n+1) \Delta x)^{p+1}-x^{p+1}\right] \\
& +\frac{1}{2}\left[(x+(n+1) \Delta x)^{p}+x^{p}\right] \\
& +\frac{1}{6} \cdot \frac{p}{2}\left[(x+(n+1) \Delta x)^{p-1}-x^{p-1}\right]
\end{aligned}
$$


268 18. Oettinger, Summenrechnung der durch einfache Functionen erzeugten Reihen.

Die Richtigkeit dieser Gleichung wird nicht aufgehoben, wenn $n-1$ statt $n$ gesetzt wird. Hierdurch wird aber eine bequemere Darstellung gefunden, und es ist

$$
\text { 354. } \quad \begin{aligned}
x^{p} & +(x+\Delta x)^{p}+(x+2 \Delta x)^{p}+\ldots+(x+n \Delta x)^{p} \\
& =\frac{1}{(p+1) \Delta x}\left[(x+n \Delta x)^{p+1}-x^{p+1}\right] \\
& +\frac{1}{2}\left[(x+n \Delta x)^{p}+x^{p}\right] \\
& +\frac{1}{6} \cdot \frac{p}{2} \Delta x\left[(x+n \Delta x)^{p-1}-x^{p-1}\right] \\
& -\frac{1}{30} \cdot \frac{1}{4} \cdot \frac{p(p-1)(p-2)}{1.2 .3}(\Delta x)^{3}\left[(x+n \Delta x)^{p-3}-x^{p-3}\right] \\
& +\frac{1}{42} \cdot \frac{1}{6} \cdot \frac{p \ldots(p-4)}{1.2 \ldots . .5}(\Delta x)^{5}\left[(x+n \Delta x)^{p-5}-x^{p-5}\right]
\end{aligned}
$$

Diese Reihe ist allgemein, und gilt für jeden Werth von $x$ und $\Delta x$, die überhaupt keiner weitern Beschränkung in ihrer Annahme unterliegen. Wir machen von ihr eine Anwendung, und setzen $x=0$ und $\Delta x=1$, dadurch entsteht der Summen-Ausdruck für die Potenzenreihe der natürlichen Zahlen:

$$
\text { 355. } \begin{aligned}
1^{p}+2^{p}+3^{p}+4^{p}+\ldots+n^{p} & =\frac{1}{p+1}\left[n^{p+1}-0^{p+1}\right] \\
& +\frac{1}{2}\left[n^{p}-0^{p}\right] \\
& +\frac{1}{6} \cdot \frac{p}{2}\left[n^{p-1}-0^{p-1}\right] \\
& -\frac{1}{30} \cdot \frac{p(p-1)(p-2)}{4.1 .2 .3}\left[n^{p-3}-0^{p-3}\right]
\end{aligned}
$$

Die Ausdrücke $0^{p+1}, 0^{p}, 0^{p-1}, \ldots$ sind dem ersten Anscheine nach überflüssig. Sie sind es aber nicht, wenn man berücksichtigt, dafs in dem Falle, wo $p-q$ in dem Ausdruck $0^{p-q}$, in Null übergeht, dieses Glied einen bestimmten Werth bekömmt. Der alsdann ist:

$$
0^{0}=\frac{\circ}{0}=1 \text {. }
$$

Benutzen wir diese Bemerkungen; so ziehen wir hieraus folgende Reihen nebst ihren Summen-Ausdrücken: 
18. Oettinger, Summenrechnung der durch einfaohe Functionen erzeugten Reihen. 269

$$
\text { 356. }\left\{\begin{array}{l}
1+2+3+4+\ldots .+n=\frac{n^{2}}{2}+\frac{n}{2}, \\
1^{2}+2^{2}+3^{2}+4^{2}+\ldots \ldots+n^{2}=\frac{n^{3}}{3}+\frac{n^{2}}{2}+\frac{n}{6}, \\
1^{3}+2^{3}+3^{3}+4^{3}+\ldots \ldots+n^{3}=\frac{n^{4}}{3}+\frac{n^{3}}{2}+\frac{n^{2}}{4}, \\
1^{4}+2^{4}+3^{4}+4^{4}+\ldots .+n^{4}=\frac{n^{5}}{5}+\frac{n^{4}}{2}+\frac{n^{3}}{3}-\frac{n}{30}, \\
1^{5}+2^{5}+3^{5}+4^{5}+\ldots .+n^{5}=\frac{n^{6}}{6}+\frac{n^{5}}{2}+\frac{5 n^{4}}{12}-\frac{n^{2}}{12}, \\
\text { u. s. w. }
\end{array}\right.
$$

Eine andere Darstellung gewinnen wir aus (354.); wenn $x=1$ gesetzt wird. Sie ist :

$$
\text { 355. } \begin{aligned}
(1+\Delta x)^{p} & +(1+2 \Delta x)^{p}+(1+3 \Delta x)^{p}+\ldots+(1+n \Delta x)^{p} \\
& =\frac{1}{(p+1)} \Delta x\left[(1+n \Delta x)^{p+1}-1\right] \\
& +\frac{1}{2}\left[(1+n \Delta x)^{p}+1\right] \\
& +\frac{1}{6} \cdot \frac{p}{2} \Delta x\left[(1+n \Delta x)^{p-1}-1\right] \\
& -\frac{1}{30} \cdot \frac{p(p-1)(p-2)}{4.1 .2 .3}\left[(1+n \Delta x)^{p-3}-1\right]
\end{aligned}
$$

Setzen wir hierin $\Delta x=2$, 80 entstehen die Potenzenreihen der ungeraden Zahlenreihen, nebst ihren Summen-Ausdrücken, und es ist:

358.

$$
\left\{\begin{array}{l}
1+3+5+7+\ldots+2 n+1=\frac{(2 n+1)^{2}}{4}+\frac{2 n+1}{2}+\frac{1}{4} \\
1+3^{2}+5^{2}+7^{2}+\ldots+(2 n+1)^{2}=\frac{(2 n+1)^{3}}{6}+\frac{(2 n+1)^{2}}{2}+\frac{2 n+1}{2}, \\
1+3^{3}+5^{3}+7^{3}+\ldots+(2 n+1)^{3}=\frac{(2 n+1)^{4}}{8}+\frac{(2 n+1)^{3}}{2}+\frac{(2 n+1)^{2}}{2}-\frac{1}{8} \\
1+3^{4}+5^{4}+7^{4}+\ldots+(2 n+1)^{4}=\frac{(2 n+1)^{5}}{10}+\frac{2 n+1^{4}}{2}+\frac{2(2 n+1)}{3}-\frac{4(2 n+1)}{15}
\end{array}\right.
$$

u. 8. w. Wird $\Delta x=3$ gesetzt, 80 gewinnt man folgende Summenreihen mit ihren Summen-Ausdrücken:

$$
\left\{\begin{array}{l}
1+4+7+10+\ldots .+3 n+1=\frac{(3 n+1)^{2}}{6}+\frac{3 n+1}{2}+\frac{1}{3} \\
1+4^{2}+7^{2}+10^{2}+\ldots .+(3 n+1)^{2}=\frac{(3 n+1)^{3}}{9}+\frac{(3 n+1)^{2}}{2}+\frac{3 n+1}{2}-\frac{1}{9} \\
1+4^{3}+7^{3}+10^{3}+\ldots .+(3 n+1)^{3}=\frac{(3 n+1)^{4}}{12}+\frac{(3 n+1)^{3}}{2}+\frac{3(3 n+1)}{4}-\frac{1}{3} \\
\text { u. s. }
\end{array}\right.
$$


270 18.- Oetting er, Summenrechnung der durch einfuche Functionen erzeugten Reihen.

§. 75.

Summirung der Potenzenreihen, deren Glieder mit abwechselnden Zeichen verbunden sind.

Um die Summen-Ausdrücke für Potenzenreihen, deren Glieder mit abwechselnden Zichen rersehen sind, zu erhalten, untersoheiden wir zwischen einer ungeraden und geraden Glieder-Anzabl.

Für die Reihe ersterer Art gilt aus (352.):

$$
X_{0}-X_{1}+X_{2} \ldots+X_{n}=\zeta^{-1} X_{n+1}+\zeta^{-1} X_{0} \text {, }
$$

für die Reihe der zweiten Art gilt aus (353.):

$$
X_{0}-X_{1}+X_{2}-X_{3} \ldots-X_{n}=-\zeta^{-1} X_{n+1}+\zeta^{-1} X_{0} \text {. }
$$

Wird hierin $X_{0}=x^{p}$ gesetzt, so ergiebt sich hieraus:

$x^{p}-(x+\Delta x)^{p}+(x+2 \Delta x)^{p}-\ldots+(x+n \Delta x)^{p}=\zeta^{-1}(x+(n+1) \Delta x)^{p}+\zeta^{-1} x^{p}$ und

$\bar{x}^{p}-(x+\Delta x)^{p}+(x+2 \Delta x)^{p}-\ldots . .-(x+n \Delta x)^{p}=-\zeta^{-1}(x+(n+1) \Delta x)^{p}+\zeta^{-1} x^{p}$. Die negativen Aufstufungen für $(x+(n+1) \Delta x)^{p}$ und $x^{p}$ erhalten wir aus

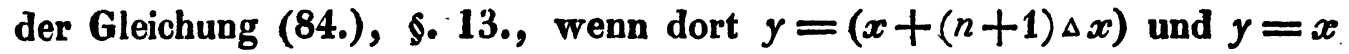
gesetzt wird. Hieraus ergiebt sich für eine Summenreihe mit ungerader Glieder - Anzahl :

360. $x^{p}-(x+\Delta x)^{p}+(x+2 \Delta x)^{p}-\ldots .+(x+n \Delta x)^{p}$

$$
\begin{aligned}
& =\frac{1}{2}(x+(n+1) \Delta x)^{p}+\frac{1}{2} x^{p} \\
& -\frac{1}{4} p(x+(n+1) \Delta x)^{p-1} \Delta x-\frac{p}{4} x^{p-1} \Delta x \\
& +\frac{1}{8} \cdot \frac{p(p-1)(p-2)}{1.2 .3}(x+(n+1) \Delta x)^{p-3}(\Delta x)^{3}+\frac{1}{8} \cdot \frac{p(p-1)}{1.2} \cdot \frac{(p-2)}{3} x^{p-3}(\Delta x)^{3}
\end{aligned}
$$

für eine Summenreihe von gerader Glieder-Anzahl aber folgende: 361. $x^{p}-(x+\Delta x)^{p}+(x+2 \Delta x)^{p}-\ldots . .(x+n \Delta x)^{p}$

$$
\begin{aligned}
& =-\frac{1}{2}(x+(n+1) \Delta x)^{p}+\frac{1}{2} x^{p} \\
& +\frac{p}{4}(x+(n+1) \Delta x)^{p-1} \Delta x-\frac{p}{4} x^{p-1} \Delta x \\
& =\frac{1}{8} \cdot \frac{\left.p^{\prime} p-1\right)(p-2)}{1.2 .3}(x+(n+1) \Delta x)^{p-3}(\Delta x)^{3}+\frac{1}{8} \cdot \frac{p(p-1)(p-2)}{1.2 .3} x^{p-3}(\Delta x)^{3}
\end{aligned}
$$

Auch hier stimmt das letzte Glied der Summenreihe der Form nach nicht mit den Gliedern der ersten Scheitelreihe im Summen-Ausdrucke überein. 
18. Oettinger; Summenrechnung der durch einfache. Functionen erzeugten Reihen. 271

Zählen wir, um Úbereinstimmung herbei zu führen, in der Gloichung (360.) $(x+(n+1) \Delta x)^{p} \mathrm{ab}$, in der Gleichung (361.) aber zu; berïcksichtigen, dals dann die Zahl der Glieder sich um eins vergröfsert, also die gerade Anzahl in die ungerade und umgekehrt ibbergeht, scheiden die gemeinschaftlichen Factoren aus, setzen $n-1$ statt $n$; so erhalten wir folgende Summen-Ausdrücke für Potenzenreihen, deren Glieder mit abwechselnden Zeichen verbunden sind, und zwar für eine gerade GliederAnzahl, aus (360.):

$$
\text { 362. } \begin{aligned}
x^{p} & -(x+\Delta x)^{p}+(x+2 \Delta x)^{p}-\ldots .-(x+n \Delta x)^{p} \\
& =-\frac{1}{2}\left[(x+n \Delta x)^{p}-x^{p}\right] \\
& -\frac{p}{4} \Delta x\left[(x+n \Delta x)^{p-1}+x^{p-1}\right] \\
& +\frac{p(p-1)(p-2)}{8.1 .2 .3}(\Delta x)^{3}\left[(x+n \Delta x)^{p-3}+x^{p-3}\right] \\
& -\frac{p(p-1) \ldots(p-4)}{4.1 .2 \ldots 5}(\Delta x)^{5}\left[(x+n \Delta x)^{p-5}+x^{p-5}\right] \\
& -\frac{17}{16} \cdot \frac{p(p-1) \ldots(p-6)}{1.2 \ldots 6}(\Delta x)^{7}\left[(x+n \Delta x)^{p-7}+x^{p-7}\right]
\end{aligned}
$$

und fuir eine Reihe von ungerader Glieder-Anzahl, aus (361.):

$$
\begin{aligned}
& \text { 363. } x^{p}-(x+\Delta x)^{p}+(x+2 \Delta x)^{p}-\ldots+(x+n \Delta x)^{p} \\
& =\frac{1}{2}\left[(x+n \Delta x)^{p}+x^{p}\right] \\
& +\frac{p}{4} \Delta x\left[(x+n \Delta x)^{p-1}-x^{p-1}\right] \\
& -\frac{1}{8} \cdot \frac{\bar{p}(p-1)(p-2)}{1.2 .3}(\Delta x)^{3}\left[(x+n \Delta x)^{p-3}-x^{p-3}\right] \\
& +\frac{1}{4} \cdot \frac{p(p-1) \ldots .(p-4)}{1.2 \ldots . .5}(\Delta x)^{5}\left[(x+n \Delta x)^{p-5}-x^{p-5}\right] \\
& -\frac{17}{16} \cdot \frac{p \ldots . .(p-6)}{1.2 \ldots . .7}(\Delta x)^{7}\left[(x+n \Delta x)^{p-7}-x^{p-7}\right] \\
& \text { ङ. } 76 .
\end{aligned}
$$

Die beiden gefundenen Gleichungen haben für die Anwendung eine sehr zweckmäfsige Gestalt. Die Grölsen $x$ und $\Delta x$ sind unabhängig von einander. Machen wir vorerst eine Anwendung auf die Potenzenreihen der natürlichen Zahlen, so haben wir $x=0$ und $\Delta x=1$ zu setzen. Dadurch versohwindet aber das erste Glied in den vorliegenden Reihen, 
272 18. Oetting er, Summenrechnurg der durch einfache Functionen erzeugten Reihen. und das zweite Glied, das mit dem negativen Zeichen versehen ist, wird zum ersten. Die Verwandlung aller Zeichen in die entgegengesetzten führt zu einer Reihe, deren erstes. Glied positiv wird: die Zahl der Glieder wird aber deswegen auch um eins verkürzt. Diese Bemerkungen führen zu folgenden Reihen, und zwar für eine ungerade Glieder-Anzahl : 364. $1^{p}-2^{p}+3^{p}-4^{p}-\ldots .+n^{p}=\frac{1}{2}\left(n_{p}-0^{p}\right)$

$$
\begin{aligned}
& +\frac{p}{4}\left(n^{p-1}+0^{p-1}\right) \\
& -\frac{1}{8} \cdot \frac{p(p-1)(p-2)}{1.2 .3}\left(n^{p-3}+0^{p-3}\right) \\
& +\frac{1}{4} \cdot \frac{p(p-1) \ldots .(p-4)}{1.2 \ldots .5}\left(n^{p-5}+0^{p-5}\right) \\
& -\frac{17}{16} \cdot \frac{p \ldots(p-6)}{1.2 \ldots . .6}\left(n^{p-7}+0^{p-7}\right)
\end{aligned}
$$

und für eine gerade Glieder-Anzahl:

365. $1^{p}-2^{p}+3^{p}-4^{p}-\ldots .-n^{p}=-\frac{1}{2}\left(n^{p}-0^{p}\right)-\frac{p}{4}\left(n^{p-1}-0^{p-1}\right)$

$$
+\frac{p(p-1)(p-2)}{8.1 .2 .3}\left(n^{p-3}-0^{p-3}\right)-\frac{1}{4} \cdot \frac{p \ldots . .(p-4)}{1.2 \ldots . .5}\left(n^{p-5}-0^{p-5}\right)
$$

Aus diesen Gleichungen entnehmen wir, mit der Berücksichtigung, dafs $0^{\circ}=\frac{\circ}{0}=1$ ist, folgende specielle Fälle für Potenzenreihen von einer ungeraden Glieder-Anzahl :

$$
\left\{\begin{array}{l}
1-2+3-4+\ldots . n=\frac{n+1}{2} \\
1-2^{2}+3^{2}-4^{2}+\ldots . n^{2}=\frac{n^{2}}{2}+\frac{n}{2}, \\
1-2^{3}+3^{3}-4^{3}+\ldots .+n^{3}=\frac{n^{3}}{2}+\frac{3 n^{2}}{4}-\frac{1}{4} \\
1-2^{4}+3^{4}-4^{4}+\ldots . n^{4}=\frac{n^{4}}{2}+n^{3}-\frac{n}{2} \\
1-2^{5}+3^{5}-4^{5}+\ldots+n^{5}=\frac{n^{5}}{2}+\frac{5 n^{4}}{4}-\frac{5 n^{2}}{4}+\frac{1}{2} \\
1-2^{6}+3^{6}-4^{6}+\ldots .+n^{6}=\frac{n^{6}}{2}+\frac{3 n^{5}}{2}-\frac{5 n^{3}}{2}+\frac{3 n}{2} \\
1-2^{7}+3^{7}-4^{7}+\ldots .+n^{7}=\frac{n^{n}}{2}+\frac{7 n^{6}}{4}-\frac{35 n^{4}}{8}+\frac{21 n}{8}-\frac{17}{8} \\
1-2^{8}+3^{8}-4^{8}+\ldots .+n^{8}=\frac{n^{8}}{2}+2 n^{7}-7 n^{6}+14 n^{3}-\frac{17 n}{2} \\
\text { u. } 8 .
\end{array}\right.
$$


18. Uet tinger, Summenrechnung der durch einfache Functionen erzeugten Reihen.

für Potenzenreihen von einer geraden Glieder-Anzahl:

$$
\text { 367. }\left\{\begin{array}{l}
1-2+3-4+\ldots \ldots-n=-\frac{n}{20} \\
1-2^{2}+3^{2}-4^{2}+\ldots . n^{2}=-\frac{n^{2}}{2}-\frac{n}{2} \\
1-2^{3}+3^{3}-4^{3}+\ldots \ldots-n^{3}=-\frac{n^{3}}{2}-\frac{3 n}{4} \\
1-2^{4}+3^{4}-4^{4}+\ldots . n^{4}=-\frac{n^{4}}{2}-n^{3}+\frac{n}{2} \\
1-2^{5}+3^{5}-4^{5}+\ldots . n^{5}=-\frac{n^{5}}{2}-\frac{5 n^{4}}{2}+\frac{5 n^{2}}{4}, \\
1-2^{6}+3^{6}-4^{6}+\ldots . n^{6}=-\frac{n^{6}}{2}-\frac{3 n^{5}}{2}+\frac{5 n^{3}}{2}-\frac{3 n}{2}, \\
\text { u. s. w. }
\end{array}\right.
$$

Setzt man in (362.) und (363.) $x=1$ und $\Delta x=2$, so gewinnt man aus ihnen die Summen fir die Potenzenreihen der ungeraden Zahlen mit abwechseinden Zeichen - , und man erbält:

$$
\text { 368. }\left\{\begin{array}{l}
1-3+5-7+\ldots .(2 n+1)=-\frac{2 n+1}{2}-\frac{1}{2}, \\
1-3^{2}+5^{2}-7^{2}+\ldots-(2 n+1)^{2}=-\frac{(2 n+1)^{2}}{2}-\frac{2 n+1}{2}-\frac{1}{2}, \\
1-3^{3}+5^{3}-7^{3}+\ldots .-(2 n+1)^{3}=-\frac{(2 n+1)^{3}}{2}-\frac{3(2 n+1)^{2}}{2}+1, \\
1-3^{4}+5^{4}-7^{4}+\ldots-(2 n+1)^{4}=-\frac{(2 n+1)^{4}}{2}-2(2 n+1)^{3}+4(2 n+1)+\frac{5}{2}, \\
1-3^{5}+5^{5}-7^{5}+\ldots-(2 n+1)^{3}=-\frac{(2 n+1)^{3}}{2}-\frac{5(2 n+1)^{4}}{2}+10(2 n+1)^{3}, \\
\text { u. s. w., },-8(2 n+1)-8,
\end{array}\right.
$$

für Potenzenreiben von ungerader Glieder-Anzahl :

369. $\left\{\begin{array}{l}1-3+5-7+\ldots .+2 n+1=\frac{2 n+1}{2}+\frac{1}{2}, \\ 1-3^{2}+5^{2}-7^{2}+\ldots+(2 n+1)^{2}=\frac{(2 n+1)^{2}}{2}+\frac{2 n+1}{2}-\frac{1}{2}, \\ 1-3^{3}+5^{3}-7^{3}+\ldots .+(2 n+1)^{3}=\frac{(2 n+1)^{3}}{2}+\frac{3(2 n+1)^{2}}{2}-1, \\ 1-3^{4}+5^{4}-7^{4}+\ldots+(2 n+1)^{4}=\frac{(2 n+1)^{4}}{2}+2(2 n+1)^{3}-4(2 n+1)+\frac{5}{2}, \\ \text { u. s. w. }\end{array}\right.$

Vergleichen wir die erste Reihe in No.356., welche die Samme der natürlichen Zahlenreihe ist, mit der zweiten Reihe in 366., welche die Unterschiede der Quadrate der natürlichen Zahlen darstellt, so haben 
274 18. Oettïng er, Summenreshnung der durch einfache Functionen erzeugten Reihen.

beide einerlei Summen-Ausdruck; folglich sind beide einander gleich, und mau bat:

$$
\text { 370. } 1+2+3+\ldots .+n=1-2^{2}+3^{2}-4^{2}+\ldots .+n^{2} \text {. }
$$

Die Summe der naturlichen Zahlen von 1 bis $n$ ist also den Unterschieden. der Quadrate derselben Zahlen, oder der Reihe der Quadrate der natülichen Zahlen, mit abwechselnden Zeichen, gleich. Diese Behauptung gilt für jedes beliebige n. Für den Fall, wo die Anzahl der Glieder in der Reihe der Quadrate gerade ist, wird das Resultat negativ.

ร. $7 \%$.

Auf dieselbe Weise, findet man auch die Summen von solchen Potenzenreihen, deren Glieder mit den fallenden Zahlen-Ausdrücken der Combinations-Classen verbunden sind. Legt man die zweite Gleichung in (350.) zu Grunde, so hat man folgende Reihe mit ihrem SummenAusdrucke:

$$
\begin{aligned}
(n+1) x^{p} & +n(x+\Delta x)^{p}+(n-1)(x+2 \Delta x)^{p}+\cdots+(x+n \Delta x)^{p} \\
& =\Delta^{-2}(x+(n+2) \Delta x)^{p}-\frac{n+1}{2} \Delta^{-1} x^{p}-\Delta^{-2}(x+\Delta x)^{p}
\end{aligned}
$$

Werden aus den Gleichungen (192.) und (193.) die entwickelten Gleichungen eingeführt, so entsteht :

371. $(n+1) x^{p}+n(x+\Delta x)^{p}+(n-1)(x+2 \Delta x)^{p}+\ldots+(x+n \Delta x)^{p}$

$$
\begin{aligned}
& =\frac{1}{(p+1)(p+2)(\Delta x)^{2}}\left[(x+(n+2) \Delta x)^{p+2}-(x+\Delta x)^{p+2}\right] \\
& -\frac{1}{(p+1) \Delta x}\left[(x+(n+2) \Delta x)^{p+1}+\frac{n+1}{1} x^{p+1}-(x+\Delta x)^{p+1}\right] \\
& +\frac{5}{12} \quad(x+(n+2) \Delta x)^{p}+\frac{n+1}{2} x^{p}-\frac{5}{12}(x+\Delta x)^{p} \\
& -\frac{p \cdot \Delta x}{12} \cdot\left[(x+(n+2) \Delta x)^{p-1}+\frac{n+1}{1} x^{p-1}-(x+\Delta x)^{p-1}\right]
\end{aligned}
$$

u. s. W.

und

Auf ähnliche Weise erhält man für die Reihen aus (351.) und (352.):

$$
\begin{gathered}
(n+1) x^{p}-n(x+\Delta x)^{p}+(n-1)(x+2 \Delta x)^{p}-\ldots+(x+n \Delta x)^{p} \\
=\zeta^{-2}(x+(n+2) \Delta x)^{p}+\frac{n+1}{1} \zeta^{-1} x^{p}+\zeta^{-2}(x+\Delta x)^{p},
\end{gathered}
$$


18. Oetting er,. Summenrechnung der durch einfache Funtionen erzeugten Reihen. 27.5

$$
\begin{aligned}
(n+1) x^{p} & -n(x+\Delta x)^{p}+(n-1)(x+2 \Delta x)^{p}-\ldots .-(x+n \Delta x)^{p} \\
& =-\zeta^{-2}(x+(n+2) \Delta x)+\frac{n+1}{1} \zeta^{-1} x^{p}+\zeta^{-2}(x+\Delta x)^{p}
\end{aligned}
$$

für die Summen - Ausdrücke für Potenzenreihen mit abwechselnden Zeichen, wenn man die angezeigten Geschäfte aus den Gleichungen (82.) und (83.) substituirt. Die Ausdrücke der Summen sind dem Anscheine nach sehr weitläufig : in der That sind sie es aber in der Anwendung auf specielle Fälle nicht, indem die zweite Scheitelreihe verschwindet, wenn $x=0$ gesetzt wird, die dritte aber nur die Einheit erzeugt, im Falle, dafs $\Delta x=1$ angenommen wird. Die weitere Ausführung überlassen wir dem Leser.

(Dio Fortsetzung folgt im nächsten Hefte.) 